\title{
Stem nourished by branches: glioblastomas co-opt classic neurotrophic factor signaling to maintain stem-like cell pool
}

\author{
Rukayat Taiwo ${ }^{1}$, Tatenda Mahlokozera ${ }^{1}$, Albert H. Kim ${ }^{1,2,3,4,5}$ \\ ${ }^{1}$ Department of Neurological Surgery, ${ }^{2}$ Siteman Cancer Center, ${ }^{3}$ Department of Neurology, ${ }^{4}$ Department of Genetics, ${ }^{5}$ Department of \\ Developmental Biology, Washington University School of Medicine, St. Louis, MO, USA \\ Correspondence to: Albert H. Kim, MD, PhD. Developmental Biology, Washington University School of Medicine, 660 S Euclid Ave., Campus Box \\ 8057, St. Louis, MO 63110, USA. Email: kima@wudosis.wustl.edu. \\ Comment on: Wang X, Prager BC, Wu Q, et al. Reciprocal Signaling between Glioblastoma Stem Cells and Differentiated Tumor Cells Promotes \\ Malignant Progression. Cell Stem Cell 2018;22:514-28.e5.
}

Received: 02 July 2018; Accepted: 11 July 2018; Published: 23 July 2018.

doi: $10.21037 /$ sci.2018.07.01

View this article at: http://dx.doi.org/10.21037/sci.2018.07.01

Glioblastoma is the most aggressive and highest grade (World Health Organization grade 4) of diffuse gliomas and is also the most common primary central nervous system malignancy in adults, accounting for $45 \%$ of brain cancers (1). Despite multimodality treatment consisting of maximal, safe, surgical resection followed by temozolomide (TMZ) chemotherapy and radiotherapy, the prognosis of glioblastoma remains challenging with a 5 -year survival rate of only $9.8 \%$ (2). Therefore, novel strategies for new glioblastoma therapies are urgently needed. Glioblastomas are characterized by significant intratumoral molecular and cellular heterogeneity (3). A major concept in the cellular heterogeneity of glioblastomas is the existence of a tumor cell hierarchy reminiscent of neural development, with a small population of self-renewing cells at the apex known as glioblastoma stem-like cells (GSCs, also called cancer stem cells or tumor-initiating cells) (4). Experimental evidence suggests that GSCs mediate radioresistance as well as tumor recurrence following TMZ treatment $(5,6)$. These observations suggest that targeting GSCs, as well as nonGSC tumor cells, may lead to more efficacious therapies.

GSCs represent a dynamic cell state, subject to cellintrinsic and -extrinsic regulation. Intrinsic regulatory mechanisms include stem cell identity-promoting genetic, epigenetic, and transcriptional programs $(7,8)$. GSCs are also subject to extrinsic regulation by paracrine signals in microenvironmental regions such as the perivascular, hypoxic, and leading edge niches. These niches provide GSCs with supportive paracrine signals such as growth factors, Notch ligands, nitric oxide, cytokines, and other cell surface and secreted factors (9-11). In turn, GSCs modulate their microenvironment to make it more tumor-permissive by secreting factors such as VEGF to drive angiogenesis and periostin to recruit M2 macrophages $(9,12)$.

An accumulating body of evidence suggests that regulators of normal neuronal functions-and synaptic transmission in particular-may modulate GSC maintenance. Brain-derived neurotrophic factor (BDNF), which is expressed and secreted by neurons in an activitydependent manner (13), regulates a wide range of neuronal activities through the engagement of the receptor tyrosine kinase TRKB (encoded by the NTRK2 gene) including survival, differentiation, synaptic plasticity, and learning and memory (14). More recently, BDNF was found to stimulate the proliferation of oligodendrocyte progenitors (OPCs) as well as GSCs $(15,16)$. But how BDNF mechanistically promotes GSC maintenance and the exact sources of BDNF in the setting of glioblastoma remained largely undefined.

In a recent study, Wang and colleagues (17) noted that GSCs secrete pro-differentiation factors including bone morphogenetic factors (BMPs) (18) as well as their inhibitors, such as gremlin 1 (GREM1) (19), leading them to hypothesize that the maintenance of a hierarchy of both stem-like and differentiated tumor cells may be essential for malignant glioblastoma tumor growth. Using flank and intracranial xenotransplantation models in immunocompromised mice, the authors first demonstrated that GSCs consistently formed tumors while differentiated glioblastoma cells (DGCs) did not. Remarkably, they observed that co-implantation of GSCs with DGCs 
accelerated tumor growth and shortened the survival of tumor-bearing mice compared to implantation of GSCs alone. Further, co-implantation of GSCs with fibroblasts did not alter either tumor growth kinetics or mouse survival, suggesting that the enhancement of tumor progression by DGCs was specific.

Reasoning that secreted factors were more likely to avail therapeutically actionable targets compared to cell-cell contact effects, the authors conducted a cytokine screen to determine the mechanisms by which DGCs modulate GSC tumorigenicity. The authors found that the neurotrophin BDNF was highly secreted by DGCs relative to matched GSCs. Analysis of datasets in the literature demonstrated that $B D N F$ mRNA is upregulated in DGCs compared to GSCs and further that this differential expression correlated with increased $B D N F$ promoter activity in DGCs as assessed by histone $\mathrm{H} 3$ lysine 27 acetylation (H3K27ac) chromatin immunoprecipitation and sequencing (ChIP-seq). Validation of these observations was performed by qPCR and ELISA assays following GSC differentiation, which revealed a sharp temporal increase in $B D N F$ mRNA expression and protein secretion in DGCs. Immunofluorescence analysis of glioblastoma surgical specimens demonstrated that $\mathrm{SOX}^{-}$, as well as astrocytic and oligodendrocyte lineage markerexpressing tumor cells co-stained for BDNF, indicating that DGCs vs. GSCs preferentially express BDNF in vivo. To strengthen the mechanistic link between BDNF secretion from DGCs and enhancement of GSC tumorigenicity, Wang et al. assessed the expression of the BDNF receptor TRKB in GSCs. TRKB mRNA was upregulated in GSCs relative to DGCs, and $\mathrm{SOX}_{2}^{+}$tumor cells co-stained for TRKB in glioblastoma surgical specimens. Strikingly, TRKB expression was elevated in GSCs when compared to nonmalignant neural cultures established from epilepsy surgical specimens, suggesting cancer-specific upregulation.

In mouse orthotopic xenotransplantation studies, the authors observed that both short hairpin RNA (shRNA)mediated knockdown and CRISPR-based knockout of $B D N F$ in DGCs attenuated their pro-tumorigenic activity when co-implanted with GSCs. Conversely, overexpression of BDNF in GSCs resulted in increased proliferation in vitro, faster tumor growth in vivo, and decreased survival of xenograft-bearing mice. These data thus implicate BDNF-TRKB signaling as a major mechanism by which DGCs potentiate GSC tumorigenicity.

The authors next hypothesized the existence of a feedforward mechanism that would propagate a paracrine circuit between DGCs and GSCs. Comparison of available
RNA-seq and H3K27ac ChIP-seq datasets revealed five genes both elevated in GSCs compared to DGCs and bearing activation marks at their promoters: key GSC transcription factors $S O X 2$ and $O L I G 2$, as well as $V G F$, $P M P 22$, and C1orf61. The authors focused on VGF (also known as VGF neural growth factor gene inducible), a neuropeptide precursor gene known to be stimulated by neurotrophin signaling in some neuronal subsets and thus a potential candidate to complete the paracrine loop between GSCs and DGCs. Intriguingly, BDNF-induced VGF was previously shown to be critical in memory and learning, including regulation of hippocampal synaptic plasticity (20).

Treatment of GSCs with BDNF in the absence of additional growth factors induced VGF expression, an effect attenuated by treatment with a pan-TRK inhibitor. Consistent with the previously described role of the PI3KAKT pathway in the induction of VGF by BDNF, AKT kinase activation also increased in GSCs following BDNF treatment. Indeed, pharmacologic inhibition of PI3K and mTOR in the presence of BDNF resulted in reduced VGF levels in GSCs.

Compared to normal brain samples in the ENCODE database, glioblastoma samples were found to exhibit highly active $V G F$ enhancers. Additionally, GSCs specifically had higher levels of H3K27ac peaks at VGF enhancers compared to that of DGCs. The authors found that serum differentiation of GSCs decreased VGF mRNA and protein levels. Acutely sorted surgical specimens revealed upregulation of VGF in $\mathrm{CD} 133^{+}$(GSC) compared to $\mathrm{CD}_{133^{-}}$(non-GSC) cells. Further, immunofluorescence assays showed that $\mathrm{SOX}^{+}$tumor cells preferentially express VGF. Supporting the concept that VGF upregulation is part of a malignant program, VGF was elevated in GSC cultures relative to neural stem cell cultures.

The authors next sought to delineate the functional autocrine and paracrine roles of GSC-derived VGF. Knockdown of $V G F$ in GSCs resulted in decreased proliferation and cell viability. Further, loss of $V G F$ reduced the stem cell identity of GSCs as shown by a reduction in the expression of stem cell markers SOX2, Nestin, and OLIG2 and in self-renewal capacity. These findings established an autocrine role for VGF in supporting GSC maintenance. To assess the paracrine function of VGF, the authors treated DGCs with the VGF-derived peptide TLQP-62, a product of post-translational processing of the precursor protein. TLQP-62 treatment in the absence of growth factors resulted in AKT activation in DGCs. Phenotypically, TLQP-62 treatment increased 
the proliferation and viability of DGCs, thus completing a BDNF-TRKB-VGF paracrine signaling circuit between DGCs and GSCs.

To directly assess the relevance of VGF to GSC tumorigenicity in vivo, the authors utilized shRNAmediated knockdown of $V G F$ in GSCs followed by orthotopic implantation into immunocompromised mice. Mice bearing $V G F$ shRNA-expressing GSCs had prolonged neurologic deficit-free survival compared to those bearing control shRNA-expressing GSCs, and remarkably, no mice harboring $V G F$ shRNA-expressing GSCs succumbed to tumors at the completion of the experiment, when all control animals had already died. Interrogation of the TCGA dataset revealed that whereas high $B D N F$ and $V G F$ expression were separately predictive of survival in a subset of patient cohorts, the combined signature of the two genes had more robust prognostic power.

One of the critical findings in this study is the demonstration that, among bona fide tumor cells, DGCs and not GSCs are a major source of the tumorsupportive neurotrophic factor BDNF, whose effect on GSC proliferation has been previously described (16). Intriguingly, there is accumulating evidence that tumorsupportive BDNF may come from microenvironmental sources. Venkatesh et al. found that, in addition to promoting tumor-supportive neuroligin-3 (NLGN3) secretion, neuronal activity increases secretion of BDNF, which demonstrated mitogenic potential in tumor cells and increased tumor cell proliferation (11). Taken together, these findings point to multiple sources of BDNF and suggest that direct inhibition of the BDNF receptor TRKB specifically in GSCs may better attenuate paracrine BDNF signaling in GSCs.

The remarkably robust effect of $V G F$ knockdown on GSC tumorigenicity across three different primary glioblastoma models is of particular interest. While the described potential mechanism of action is PI3KAKT activation, it is possible that VGF may have asof-yet undefined autocrine roles in promoting GSC tumorigenicity. Indeed, the exact identity of GSCpromoting peptides generated by VGF processing and the receptor(s) bound by VGF-derived peptides in GSCs have not yet been identified. This finding of an in vivo dependence of high-grade gliomas (HGG) on secreted factors implicated in synaptic function and plasticity is reminiscent of a similar phenomenon observed with NLGN3, a neuronal cell-surface protein that is a critical mediator of synapse formation and remodeling (21).
Venkatesh et al. (22) found that HGG lines, including adult glioblastoma, were severely inhibited in tumorigenic capacity when implanted into NLGN3 knockout mice. They further showed that microenviromental NLGN3 released from active neurons induces focal adhesion kinase (FAK) phosphorylation and subsequent activation of the SRC kinase, PI3K-mTOR, and RAF-MEK-ERK kinase cascades. Additionally, NLGN3 signaling results in feed-forward expression of NLGN3 in glioma cells. Similar to VGF, the receptor bound by NLGN3 on glioma cells is yet to be elucidated. Future studies to investigate potential crosstalk between VGF and NLGN3 signaling and the possibility of therapeutic dual targeting of these pathways in glioblastoma are warranted.

The fact that neuronal synaptic regulatory molecules (BDNF, VGF, and NLGN3) in glioblastoma seem to converge on key oncogenic nodes, specifically the PI3KAKT pathway $(17,22)$, raises some concerns for both heterogeneous intratumoral responses and acquisition of resistance to therapies targeting these molecules. Alterations in the RTK/RAS/PI3K pathway occur in up to $88 \%$ of glioblastomas, increasing the likelihood of downstream resistance to inhibition of oncogenic signaling initiated by paracrine synaptic function modulators in GSCs (23). Further, spatial heterogeneity in the occurrence and frequency of copy number alterations and mutations in the RTK/RAS/PI3K pathway have been described $(24,25)$, suggesting that subclones within the same tumor may display divergent responses to targeted inhibitors of neurotrophin and other synaptic regulator-dependent signaling. These considerations should motivate further research into other possible mechanisms of action of the BDNF-TRKB-VGF circuit in glioblastoma so that multi-level therapies in combination might be utilized to counteract potential therapeutic resistance.

\section{Acknowledgments}

Funding: This work was supported by National Institutes of Health Grant R01 NS094670, the Christopher Davidson and Knight Family Fund (A.H.K.), the Howard Hughes Medical Institute Medical Fellows Program (R.T.), and the Washington University Medical Scientist Training Program grant NIH T32 GM07200 (T.M.).

\section{Footnote}

Conflicts of Interest: The authors have no conflicts of interest 
to declare.

\section{References}

1. Ostrom QT, Gittleman H, Liao P, et al. CBTRUS Statistical Report: Primary brain and other central nervous system tumors diagnosed in the United States in 20102014. Neuro Oncol 2017;19:v1-v88.

2. Stupp R, Hegi ME, Mason WP, et al. Effects of radiotherapy with concomitant and adjuvant temozolomide versus radiotherapy alone on survival in glioblastoma in a randomised phase III study: 5-year analysis of the EORTC-NCIC trial. Lancet Oncol 2009;10:459-66.

3. Aum DJ, Kim DH, Beaumont TL, et al. Molecular and cellular heterogeneity: the hallmark of glioblastoma. Neurosurg Focus 2014;37:E11.

4. Singh SK, Hawkins C, Clarke ID, et al. Identification of human brain tumour initiating cells. Nature 2004;432:396-401.

5. Bao $\mathrm{S}, \mathrm{Wu} \mathrm{Q}, \mathrm{McLendon} \mathrm{RE}$, et al. Glioma stem cells promote radioresistance by preferential activation of the DNA damage response. Nature 2006;444:756-60.

6. Chen J, Li Y, Yu TS, et al. A restricted cell population propagates glioblastoma growth after chemotherapy. Nature 2012;488:522-6.

7. Suva ML, Rheinbay E, Gillespie SM, et al. Reconstructing and reprogramming the tumor-propagating potential of glioblastoma stem-like cells. Cell 2014;157:580-94.

8. Zheng H, Ying H, Yan H, et al. p53 and Pten control neural and glioma stem/progenitor cell renewal and differentiation. Nature 2008;455:1129-33.

9. Lathia JD, Heddleston JM, Venere M, et al. Deadly teamwork: neural cancer stem cells and the tumor microenvironment. Cell Stem Cell 2011;8:482-5.

10. Qin EY, Cooper DD, Abbott KL, et al. Neural PrecursorDerived Pleiotrophin Mediates Subventricular Zone Invasion by Glioma. Cell 2017;170:845-59.e19.

11. Venkatesh HS, Johung TB, Caretti V, et al. Neuronal Activity Promotes Glioma Growth through Neuroligin-3 Secretion. Cell 2015;161:803-16.

12. Zhou W, Ke SQ, Huang Z, et al. Periostin secreted by glioblastoma stem cells recruits M2 tumour-associated macrophages and promotes malignant growth. Nat Cell Biol 2015;17:170-82.

13. Hong EJ, McCord AE, Greenberg ME. A biological function for the neuronal activity-dependent component of Bdnf transcription in the development of cortical inhibition. Neuron 2008;60:610-24.

14. Lee FS, Kim AH, Khursigara G, et al. The uniqueness of being a neurotrophin receptor. Curr Opin Neurobiol 2001;11:281-6.

15. Tsiperson V, Huang Y, Bagayogo I, et al. Brain-derived neurotrophic factor deficiency restricts proliferation of oligodendrocyte progenitors following cuprizone-induced demyelination. ASN Neuro 2015;7.

16. Lawn S, Krishna N, Pisklakova A, et al. Neurotrophin signaling via $\mathrm{TrkB}$ and $\mathrm{TrkC}$ receptors promotes the growth of brain tumor-initiating cells. J Biol Chem 2015;290:3814-24.

17. Wang X, Prager BC, Wu Q, et al. Reciprocal Signaling between Glioblastoma Stem Cells and Differentiated Tumor Cells Promotes Malignant Progression. Cell Stem Cell 2018;22:514-28.e5.

18. Piccirillo SG, Reynolds BA, Zanetti N, et al. Bone morphogenetic proteins inhibit the tumorigenic potential of human brain tumour-initiating cells. Nature 2006;444:761-5.

19. Yan K, Wu Q, Yan DH, et al. Glioma cancer stem cells secrete Gremlin1 to promote their maintenance within the tumor hierarchy. Genes Dev 2014;28:1085-100.

20. Alder J, Thakker-Varia S, Bangasser DA, et al. Brainderived neurotrophic factor-induced gene expression reveals novel actions of VGF in hippocampal synaptic plasticity. J Neurosci 2003;23:10800-8.

21. Sudhof TC. Neuroligins and neurexins link synaptic function to cognitive disease. Nature 2008;455:903-11.

22. Venkatesh HS, Tam LT, Woo PJ, et al. Targeting neuronal activity-regulated neuroligin-3 dependency in high-grade glioma. Nature 2017;549:533-7.

23. Cancer Genome Atlas Research N. Comprehensive genomic characterization defines human glioblastoma genes and core pathways. Nature 2008;455:1061-8.

24. Sottoriva A, Spiteri I, Piccirillo SG, et al. Intratumor heterogeneity in human glioblastoma reflects cancer evolutionary dynamics. Proc Natl Acad Sci U S A 2013;110:4009-14.

25. Mahlokozera T, Vellimana AK, Li T, et al. Biological and therapeutic implications of multisector sequencing in newly diagnosed glioblastoma. Neuro Oncol 2018;20:472-83.

doi: 10.21037/sci.2018.07.01

Cite this article as: Taiwo R, Mahlokozera T, Kim AH. Stem nourished by branches: glioblastomas co-opt classic neurotrophic factor signaling to maintain stem-like cell pool. Stem Cell Investig 2018;5:22. 